TRANS $\cdot$ núm. I $5 \cdot 201 \mathrm{I}$

DOSSIER $\cdot 69-82$
Este artículo tiene como objetivo explorar las razones que motivan a los jugadores de videojuegos para invertir cientos de horas de ocio en decodificar, coordinar y traducir juegos para la comunidad de aficionados. Se investiga, por tanto, cómo se organizan los proyectos y a qué aspectos de la traducción dan más importancia tanto los traductores como quienes usan sus parches. Como parte de la labor de investigación, la autora se puso en contacto con ocho de los romhackers más destacados de habla hispana: seis españoles, un argentino y un costarricense. En este artículo se plasman las opiniones, comentarios e impresiones que compartieron por correspondencia y se analiza la situación de esta práctica que sigue de rabiosa actualidad.

PALABRAS CLAVE: localización de videojuegos, traducción de videojuegos, romhacking, traducción amateur

\title{
La traducción amateur de videojuegos al español
}

\section{Diana Díaz Montón}

Wordlab Translation $\&$ Localisation Services, Founder

\section{Amateur Videogame Translation into Spanish}

The goal of this article is to explore the reasons that drive videogame players to spend hundreds of hours of their free time to decode, coordinate and translate videogames for the fan community. The article analyses how projects are organised and what aspects of the translation are most important for both the translators and those who download their patches. As part of the research, the author got in touch with eight of the most prominent Spanish-speaking romhackers. This article is a compilation of their opinions, comments and impressions and evaluates the current situation of this practice that is still very much in trend.

KEY WORDS: video game localization, video game translation, romhacking, fan translation, amateur translation. 


\section{LA TRADUCCIÓN AMATEUR Y EL ROMHACKING}

La traducción amateur o fan translation de videojuegos es una práctica que cobró gran popularidad a finales de los años noventa con la proliferación de los «emuladores»: programas que ejecutan la ROM de juegos para poder volver a disfrutar en PC títulos de las consolas y equipos obsoletos, cuyo funcionamiento imitan mediante una máquina virtual por software. Los jugadores recuperaron el interés por los clásicos de su infancia y surgió un movimiento para traducir títulos que no se habían traducido o que sencillamente no se llegaron a comercializar en ciertas regiones. Para extraer los textos que tanto deseaban trasladar a sus respectivos idiomas era necesario modificar la ROM, la memoria de solo lectura del juego. La generación de los emuladores contaba por suerte con los conocimientos y técnicas necesarios para ello. Es lo que se conoce como rombacking. El rombacking es pues el proceso por el que se altera la ROM con el fin de modificar los distintos elementos de que se compone, desde los textos y gráficos, a la arquitectura (escenarios) y dinámica de juego. Aunque a menudo se habla de hacking, con connotaciones negativas (asociado a prácticas de manipulación ilegales, porque el software no está diseñado para que pueda ser modificado por el usuario), en este artículo veremos que el modo en que se utiliza en la localización de videojuegos parece ser no solo positivo sino beneficioso, ya que extiende la popularidad de estos juegos más allá de la distribución oficial.

Como apunta Muñoz Sánchez (2008), para introducir cambios en la ROM es preciso recurrir a técnicas de ingeniería inversa. Los rombackers emplean editores hexadecimales (programas informáticos que permiten modificar los archivos binarios) y herramientas especializadas, muchas de factura propia, para extraer, editar y volver a integrar los contenidos y elementos modificados. Si bien una vez se tiene acceso a la ROM ésta se podría modificar en su totalidad, la realidad es que esta actividad suele centrarse casi en exclusiva en la traducción, y como mucho se alteran algunos elementos gráficos con el único fin de que los textos se lean con claridad y sin cortes. Una vez finalizado el trabajo de localización, los rombackers distribuyen un parche con los nuevos contenidos para instalar sobre el juego original. Como nota curiosa, aunque no exenta de cierto matiz preocupante, existe un movimiento derivado de la traducción amateur conocido como undubbing que consiste en modificar los contenidos de juegos doblados para recuperar el audio original, manteniendo los subtítulos localizados. La comunidad de jugadores es especialmente crítica con el doblaje y no suele encajar bien las entonaciones sin sentido ni las voces que se repiten excesivamente. Muchos prefieren escuchar a un reparto de actores al que consideran de mayor calidad simplemente porque está compuesto por estrellas del cine o la televisión, pero conservan los subtítulos localizados porque el conocimiento del idioma original no es tan elevado, sobre todo si se trata de obras en japonés.

\section{LA ESCENA $A M A T E U R$ DE HABLA HISPANA}

La traducción amateur de videojuegos es una práctica muy generalizada y por tanto no es exclusiva de un idioma, región o país. La comunidad angloparlante lleva haciéndolo, desde el japonés, desde principios de los años 90, si nos atenemos a los datos de Wikipedia (<http://en.wikipedia.org/wiki/Fan_translation_of_video_games $>$ ). $Y$ aunque es difícil saber cuál fue la primera traducción amateur de 
un videojuego al castellano, puesto que muchos rombackers trabajan en solitario y distribuyen sus parches en sitios web, foros y blogs que no siempre perduran, lo cierto es que la comunidad hispana está en plena efervescencia. Actualmente el sitio web de referencia es Romhack Hispano, un dominio que da alojamiento a gran parte de la comunidad hispana y sus respectivos proyectos, tanto individuales, como de grupo. En total agrupa 15 sitios que suman decenas de parches con traducciones para todas las consolas, desde la primera PlayStation, de las más populares, a viejas glorias como la Super NES y la MegaDrive, además del sempiterno PC. Los rombackers hispanohablantes se cuentan por miles, pero el núcleo duro, los más activos y normalmente encargados de gestionar grandes proyectos, no llegarán a una docena. Los sitios más activos son, a día de hoy, Fortaleza Romhack y Tales Translations, volcados estos últimos en la serie japonesa Tales, de los que hablaremos más adelante.

Fuera del foro y el dominio Romhack Hispano, otro de los grupos más atareados es el Clan DLAN, que además de traducir también crea mods, esto es, modificaciones para «hacer más amenas vuestras aventuras», según reza el propio sitio web. Este grupo es también muy fan de los juegos de rol (RPG por sus siglas en inglés), uno de los géneros que cuenta con mayor número de seguidores entre los rombackers, y en su Academia de Sundabar ofrecen traducciones propias de títulos como Thief y System Shock 2; sagas como Elder Scrolls y Neverwinter Nights, e incluso traducciones mejoradas, como la de Dragon Age, y de comunidades amigas. Según anuncian son más de 700 las traducciones recopiladas.

Si bien la comunidad agrupa a rombackers de todas las nacionalidades, los españoles predominan en la escena y gozan de gran populari-
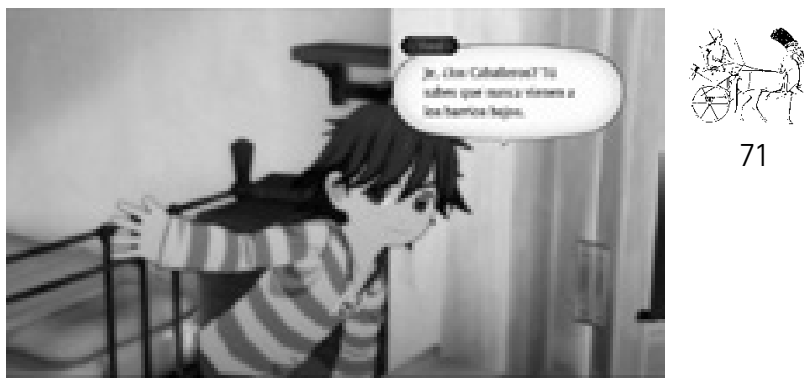

Figura I. Tales of Vesperia, de Tales Translations (<bttp:// notas.tales-tra.com/img/20Io/og/amerigo-bienI-69ox388. $j p g>$ )

dad y respeto entre sus colegas de países como Argentina, Chile o Costa Rica. Son el grupo más nutrido y longevo y han contribuido a enriquecer el panorama con todo tipo de tutoriales y utilidades para que otros puedan aprender. Pero como indica Reinhardt, «el rombacking es muy internacional», $y$ suelen reunirse en foros de otros países no hispanohablantes para intercambiar información sobre distintos programas, métodos, etcétera.

\section{ROMHACKER: PERFIL Y MOTIVACIONES}

Los ocho rombackers que respondieron a la breve encuesta que les envié por correo electrónico llevan modificando ROM y traduciendo juegos de forma más o menos constante en una horquilla que oscila entre cuatro y diez años, cuando empezaban a circular hacks por Internet y proliferaban los tutoriales de cómo modificar las ROM, en el apogeo de la emulación que comentábamos anteriormente. Los más veteranos se remontan incluso a la época de la Super NEs, la consola de principios de los noventa con la que crecieron los pioneros de la localización amateur. Como apunta Wastor en sus respuestas a mi entrevista, con los años llegan las responsabilidades — trabajo, familia- y les queda menos tiempo para dedicarse 
a los proyectos. Este destaca además la labor del rombacker frente a quienes se dedican a traducir únicamente. Son ellos quienes hacen posible la traducción amateur, pues gracias a sus conocimientos de ingeniería, entienden cómo funciona el juego y saben modificarlo. Pero hace falta un relevo generacional, nuevos fans que hereden los conocimientos de los actuales rombackers y que puede que incluso lleguen a dar el salto al mundo profesional, como Pablito's que, tras su paso por Nintendo of Europe, ahora trabaja como traductor autónomo. En solitario y en grupo han participado en una media de tres juegos completos liberados. Algunos proyectos se quedaron por el camino, otros avanzan con parsimonia y casi todos los rombackers tienen en casa una colección enorme de videojuegos, así como gran cantidad de pequeñas traducciones e infinidad de pruebas.

Pero, ¿qué mueve a miles de jugadores a dedicar sus horas de ocio a investigar el código de programación del videojuego y traducir interminables diálogos? La respuesta es prácticamente unánime: la posibilidad de disfrutar «en condiciones». Y aunque en origen lo hacen para sí mismos, porque se divierten investigando el código y peleándose con los «scripts», como apuntan los miembros del Clan DLAN, a la mayoría les entristece que quienes desconocen el idioma no puedan jugar a «grandes títulos» porque no entienden los diálogos. Para ellos, poder llevar ese disfrute a otros jugadores es, en sus propias palabras, un orgullo y un placer; sienten que todo el esfuerzo «sirve para algo». La expectación que despiertan las traducciones que los fans esperan como agua de mayo y el clamor popular son un potente motor y una fantástica recompensa a todo el trabajo. También hablan de la traducción en términos de reto, un pasatiempo entretenido, una forma de promover su lengua materna y de aprender y mejorar el inglés (o japonés para quien se atreve con este idioma). Pese a que cada vez se localizan más títulos al castellano, el hecho de que la comunidad hispana de rombackers siga tan activa y surjan siempre nuevas propuestas y peticiones de traducción demuestra que queda todavía mucha demanda por cubrir por parte de desarrolladores y distribuidoras en términos de localización. Por lo que se desprende de las entrevistas y de los textos con que se presentan los distintos colectivos de traducción amateur, preferirían que las distribuidoras comercializaran los juegos localizados. Son conscientes del esfuerzo y el coste que acompaña a las traducciones oficiales y aunque a veces lanzan críticas encendidas contra estas, cada vez se muestran más comprensivos con los errores de localización y enfocan siempre las retraducciones como un servicio a la comunidad. A la hora de elegir entre un título u otro, la gran mayoría no distingue entre nuevo y antiguo, y anteponen la calidad del juego a su fecha de lanzamiento o la plataforma para la que se creó. Muchos se decantan por juegos antiguos porque tienen un significado personal para ellos y traducirlos y ofrecer el parche a otros fans es una forma de revivir y compartir recuerdos gratos. Aunque lo cierto es que un título nuevo genera una gran expectación y si se embarcan en estos proyectos saben que llegarán a más gente, lo cual es un gran aliciente.

\section{EQUIPO Y PROCESOS DE TRABAJO}

En origen, el trabajo de los traductores e ingenieros aficionados es bastante individualista $\mathrm{y}$ se concentran en una misma figura todas las tareas, desde la extracción hasta el testeo del parche. Al no existir plazos ni presiones externas para el lanzamiento, no es descabellado que un jugador decida ponerse manos a la obra en 
solitario y logre finalizar su objetivo. Con la proliferación de sitios web y foros de traducción amateur, cada vez es más habitual que un jugador tome la iniciativa y dé a conocer su idea de traducir tal o cual título y busque apoyo en otros compañeros. Bien porque necesita ayuda para las tareas de rombacking previamente dichas (extracción, compilación, modificación de gráficos, fuentes, etcétera), bien porque el volumen de texto es demasiado grande para una sola persona. Los colectivos como Romhack Hispano y Clan DLAN son plataformas que facilitan la comunicación entre los rombackers y son el punto de encuentro entre éstos y el resto de la comunidad de jugadores que utilizan sus traducciones. No existen grupos de rombackers rígidos, sino que suelen formarse por proyecto y se disuelven una vez finalizada la traducción, si bien muchos vuelven a encontrarse y gracias a los foros la colaboración es abierta y todos participan en múltiples conversaciones. Clan DLAN es la comunidad más amplia y cuenta con unos 15.000 miembros, según sus administradores. Los proyectos surgen de forma espontánea cuando un usuario escribe una entrada en el foro para pedir ayuda o proponer una traducción. Como apunta Bragolsûl en una entrevista concedida a la publicación online Último Nivel (<http://www.ultimonivel. net/2009/r2/entrevista-a-clandlan/>), lo ideal es que haya un coordinador, que se fije una cantidad de traductores adecuada para el proyecto y se cuente con testers y programadores para las tareas más técnicas de extracción de textos y su recodificación en lugar de concentrarlas todas en una sola persona.

Otro grupo muy interesante es Tales Translations, un colectivo que surgió de su devoción a la serie «Tales» de Namco (ahora Namco Bandai). Comenzaron su andadura en 2006, año en que se lanzó Tales of Eternia en España en su versión para PSP (el original para PlayStation data de 2000). El grueso de la traducción lo realizaron tres personas y contaron puntualmente con la colaboración de hasta veinte fans más. En la web del proyecto recalcan su esfuerzo por conseguir una traducción de calidad, revisada y testeada, fiel incluso a las traducciones oficiales de otras entregas. Es una de las comunidades más activas y mejor organizadas, con micrositios dedicados a cada proyecto donde además de distribuir los parches con la traducción, incluyen anécdotas, instrucciones para instalar los textos y hasta notas donde detallan los entresijos de su trabajo, salpicado todo de un humor muy informal que conecta perfectamente con los jugadores. Recientemente han lanzado la traducción del Tales of Destiny, que les ha llevado tres años y tienen en marcha otros dos «Tales». En las tabla I se muestra el volumen de trabajo y la dedicación de estos grupos de traductores e ingenieros amateur.

Su experiencia en estos años les ha hecho perfeccionar el método de trabajo. Cuentan en su web que para Tales of Vesperia, uno de los títulos en los que están trabajando, dedicaron un par de semanas a redactar recomendaciones para la traducción, elaborar glosarios de términos y definir una metodología con el fin de «traducir y revisar con más calidad y ofrecer un «producto» del que podamos estar muy orgullosos». En su llamamiento para reclutar a nuevos colaboradores (traductores y revisores), enumeran una serie de requisitos e indican que harán una prueba de traducción a quienes se interesen por el proyecto. Buscan voluntarios con un buen dominio del español y buen nivel de inglés, con experiencia previa y, lógicamente, mucho tiempo libre y ganas de trabajar.

Los integrantes del Chrono sp Team, que se formó para traducir Chrono Trigger para Nintendo DS, también consideraron varios 
Tabla i. Cifras de la localización de Tales of the Abyss, por Tales Translations

\begin{tabular}{|c|c|}
\hline TÍTULO & Tales of the Abyss \\
\hline DESARROLLADORA & Namco Tales Studio \\
\hline PLATAFORMA & $\mathrm{PS}_{2}$ \\
\hline LANZAMIENTO & Japón: 2005, EE.UU.: 2006, no publicado en España \\
\hline MICROSITIO & http://toa.tales-tra.com/?s=main \\
\hline VOLUMEN & 340.000 palabras \\
\hline PLAZO DE EJECUCIÓN & I,5 años \\
\hline EQUIPO & I3 personas (9 traductores +4 testers) \\
\hline TAREAS & $\begin{array}{l}\text { programación, programación de utilidades, ingeniería inversa, edición gráfica, } \\
\text { subtitulado de vídeos, capturas de manual, soporte logístico, diseño web, admi- } \\
\text { nistración web, administración foro, maquetación, traducción, revisión, testeo }\end{array}$ \\
\hline
\end{tabular}

(<http://blog.tales-tra.com/2009/noticias/sobre-la-traduccion-de-tales-of-vesperia/>)

métodos de trabajo. Finalmente adoptaron el que propuso Reinhardt: distribuyeron entre los miembros el listado de archivos y cada uno fue eligiendo los que quería hacer. Una vez terminados, se volvían a publicar los archivos o scripts para su revisión y testeo. Cuando consideraban que estaba todo bien, lo daban por cerrado. En un primer momento dividieron los scripts por personajes, de forma que cada traductor se encargaba de uno y así se aseguraban de que los parlamentos conservasen un mismo tono y estilo. Por falta de personal y de constancia de algunos de los voluntarios tuvieron que renunciar a este método que dejaba muchos archivos a medias, pero al menos el estilo de cada personaje ya se había marcado y les resultó sencillo mantenerlo.

Para mantener a la comunidad informada y propiciar la colaboración de más voluntarios, suelen publicar en los foros y sitios web calendarios de los proyectos con el progreso del mismo (fig. 2).
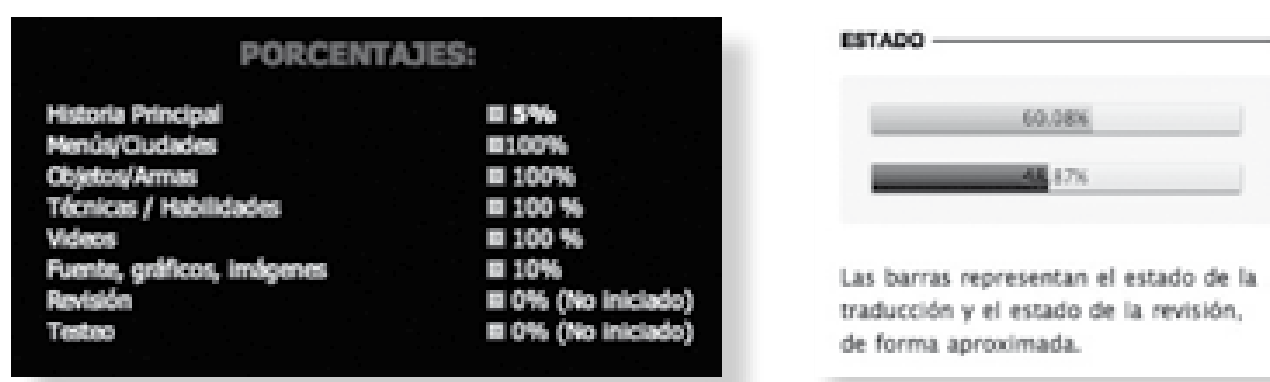

Las barras representan el estado de la traduceide y el escado de la revision, de forma aprovimada.

Figura 2. Progreso de la traducción de Lunar Silver Star Harmony y Tales of Vesperia (<http://devil.romhackhispano.org/ lunar.html $>)(<$ bttp://blog.tales-tra.com/proyectos/tales-of-vesperia/ $>)$ 
En total invirtieron unos ocho meses de trabajo entre la traducción y la fase final de testeo, que tampoco descuidaron: el hecho de que se «pasaran» el juego veinte veces completas para buscar fallos, cuando las compañías profesionales de localización de videojuegos suelen realizar entre tres y cinco ciclos de testeo, da fe de su cariño y respeto por el juego en sí.

\section{LAS TRES C: COMPRENSIÓN, CORRECCIÓN, CREATIVIDAD}

Cuando se pregunta a los rombackers a qué aspectos de la traducción dan más importancia, casi todos coinciden en la corrección; no conciben una traducción con errores gramaticales y se empeñan en que estén disponibles todos los caracteres especiales que emplea el español («ñn, tildes), de ahí que se modifiquen las fuentes o las tipografías para añadir caracteres y que el texto se muestre debidamente en pantalla. Quienes, por falta de medios o conocimientos, no logran modificar las fuentes para que aparezcan los acentos o los signos de exclamación o interrogación de apertura y cierre, tienen que renunciar a ellos, lo cual empaña el resultado. La mayoría habla de forma casi instintiva de ortotipogafía, que se mantenga la personalidad del personaje, que la traducción no sea literal, que esté adaptada al idioma, que se empleen modismos y se respeten las palabras clave. Reinhardt, por ejemplo, afirma que un buen traductor debe dar importancia a todo, si algún aspecto «cojea», el conjunto se ve afectado y el usuario lo nota. Dice también «necesito que todo sea perfecto», lo cual es una prueba del valor de la traducción para la comunidad de jugadores y de que detrás de esta dedicación se encuentra la mejor de las intenciones. En Fortaleza Romhack se pueden encontrar declaraciones similares; disponen de una sección específica dedicada a la interpretación del juego. Según ellos, es importante saber transmitir la idea original de la forma más fiel posible: estados de ánimo, emociones, nombres propios, que se mantenga la historia sin confundir al jugador. Es importante adaptar expresiones y mantener la forma de ser y de hablar del personaje. PacoChan de TT y Soywiz añaden además que los textos estén bien escritos. Una traducción con faltas y sin acentos no es «bonita», no se puede tomar en serio. Si la redacción es mala levanta dudas sobre la precisión de la
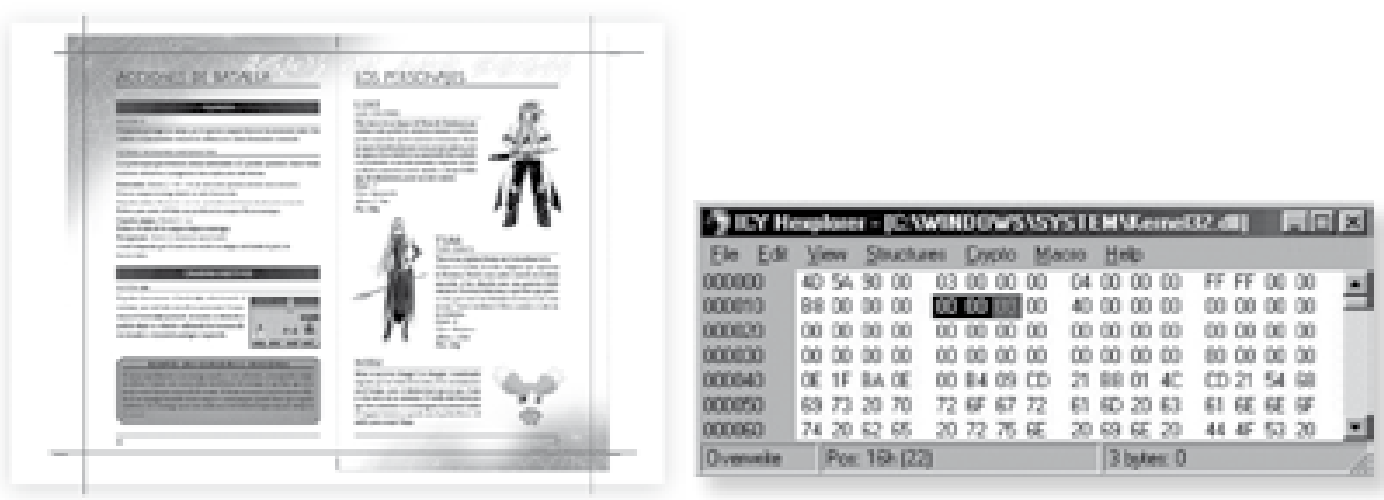

Figura 3. Manual de Tales of the Abyss y ejemplo de editor hexadecimal (<http://toa.tales-tra.com/i/manual-explicacion-3. png ) (<http://okami.rombackhispano.org/img/translhexion.jpg $\rangle)$ 
traducción.

Soywiz se centra más en las labores propias del rombacking. Para él, uno de los aspectos clave es eliminar las barreras que presenta el código, no preparado para ser localizado. Hablamos de la limitación de espacio, la ausencia de caracteres especiales, o la posibilidad de preparar y extraer el texto para que el grupo pueda trabajar cómodamente y centrarse en la traducción sin necesidad de contar con conocimientos técnicos. Curiosamente este tipo de estrategia es el que recientemente desarrolladores y distribuidores de videojuegos están tratando de implementar.

\section{Al otro lado del espejo}

Pero, ¿los jugadores valoran los mismos aspectos? ¿Son críticos con el resultado? Como bien apunta DaRKWizaRD lo primero es la comprensión: «Quien busca una traducción es porque no entiende el idioma de origen del juego y una traducción que falla en ese aspecto no soluciona del todo ese problema.» Traducciones que sean sencillas de leer y sin errores garrafales. La corrección y la creatividad quedan pues relegadas a un segundo plano; de hecho el umbral de exigencia es bastante bajo. En cuanto al aspecto creativo hay disparidad de pareceres. Por un lado es difícil contentar a toda la comunidad hispana; los rombackers españoles son los más numerosos y es habitual que utilicen modismos locales que no se entienden ni gustan en otros países hispanohablantes, lo cual a veces provoca roces. De hecho, en los sitios web de algunos proyectos se incluyen notas al respecto y avisan de que su traducción incluye modismos. Con mayor o menor tino vienen a decir que a fin de cuentas se trata de un trabajo no oficial, que han hecho como les ha apetecido y al que no le guste la traducción, que no la utilice. Por otro lado, algunos ven con buenos

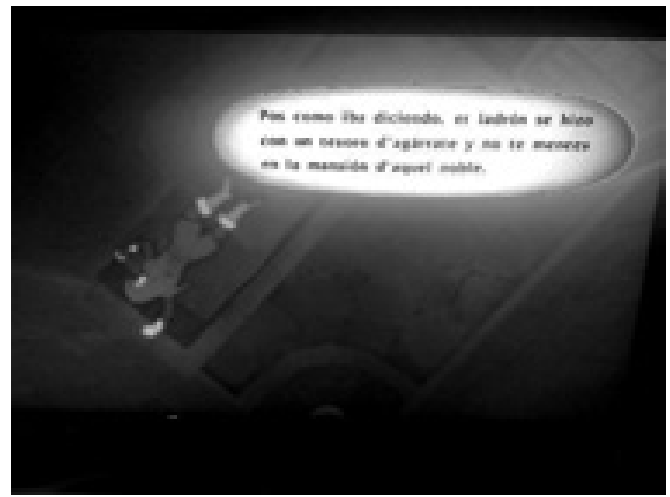

Figura 4. Coloquialismos en el Tales of Vesperia (<http:// notas.tales-tra.com/img/20I0/og/IMG_oo94.jpg $\rangle$ )

ojos que los traductores adapten chistes, bromas o rimas, pero la gran mayoría, al conocer el juego en su versión original, prefiere que se respeten los nombres y no son muy bienvenidas las adaptaciones de nombres personajes, lugares, objetos, etcétera.

Soywiz vuelve a insistir en que lo ideal es que el conjunto esté equilibrado, empezando primero por una traducción correcta, que no sea literal, que resulte amena, donde se muestren todos los caracteres especiales y no haya faltas de ortografía. Y en cuanto a la creatividad, añade que si bien no es una de las características a priori más valoradas por los jugadores, al menos no de una forma consciente, es lo que afecta más directamente a la diversión. La inmersión total es fundamental para disfrutar. La comunidad de jugadores es más benévola con las traducciones amateur, pues entienden que es una labor compleja y desinteresada, pero si el resultado es «cutre», si faltan espacios, la puntuación es incorrecta o el texto está salpicado de faltas de ortografía, no dudan en quejarse. Las buenas traducciones se comentan pero, como en todo, las malas se comentan aún más. Los baremos son distintos y el umbral es más 
bajo que el que marcan los propios rombackers, pero sí saben apreciar las buenas traducciones y no tienen pelos en la lengua a la hora de criticar las malas.

\section{PROFESIONALES VS. AMATEUR}

La metodología que aplica por norma general la comunidad de rombackers guarda muchos paralelismos con la que se emplea en el ámbito profesional, llegando incluso a superarla en según qué casos, sobre todo por los errores que se detectan en traducciones profesionales, debido a la falta de contexto, información, tiempo y comunicación con los traductores. Un ejemplo bastante conocido entre los jugadores es el de la traducción de "piece of cake» por «trozo de tarta» en Elder Scrolls IV: Oblivion, cuando en realidad el inglés se refería a la frase hecha para expresar que algo es muy sencillo, algo así como "pan comido» o «coser y cantar». El error vino por incluir "piece of cake» en una larga lista de términos para el glosario, sin contexto alguno, cuando esta expresión no era realmente un término y por tanto no se hacía necesario incluirla en el glosario. Su inclusión habría estado justificada de haberse tratado de una coletilla que repitiese constantemente un personaje, por ejemplo, y se pretendiese que siempre se tradujera igual. Este fallo, si hubiera aparecido el contexto, no se habría producido, y al jugador le descoloca bastante escuchar a uno de los personajes decir «un trozo de tarta» sin venir a cuento.

Los procesos de trabajo se han perfeccionado de forma natural y están basados en la experiencia y el sentido común. Además, la traducción amateur cuenta con cuatro ventajas indiscutibles: la flexibilidad de los plazos, la motivación, el conocimiento del juego y su entrega a la comunidad de jugadores. Sin duda, las claves de su éxito. Cuando se embarcan en un nuevo trabajo, los traductores amateur disponen de una copia del juego que van a modificar y están de sobra familiarizados con él, un lujo del que los traductores profesionales no gozan a menos que trabajen en plantilla en el departamento de localización de una desarrolladora, y a veces ni eso. En la localización amateur de Tales of the Abyss el scripts también lo tradujeron a ciegas, no sabían a qué personaje correspondía cada diálogo y aunque echaron mano de memoria, como indican en sus notas la clave fue una vez más el testeo. Y añaden: «en la mayoría de traducciones oficiales están en las mismas y por eso se tienen que realizar exhaustivísimos testeos.»

Como en la escena profesional, el equipo y los plazos dependen de la envergadura del proyecto. Aunque a priori puede dar la sensación de que la traducción amateur es más descontrolada, porque colabora más gente, en lo profesional, si bien lo ideal es que el número de traductores no se altere durante el proyecto y el grupo no cambie, lo cierto es que no siempre sucede así. No es extraño que los términos del proyecto varíen si, por ejemplo, el volumen de palabras es mayor de lo previsto y hay que ampliar el equipo. Estas nuevas incorporaciones que se suman al proyecto ya empezado no tienen el mismo grado de familiarización ni el mismo rodaje que el resto de los integrantes. Tampoco es extraño que si la localización se divide en fases, con semanas e incluso meses de diferencia entre una y otra, algunos traductores no estén disponibles y haya que recurrir a otros nuevos, luego la estabilidad del equipo tampoco está garantizada en el plano profesional. Para hacer frente a esos cambios, los departamentos y empresas de localización utilizan guías de estilo; herramientas de traducción asistida; se define la forma en que se va a abordar un per- 
sonaje; se emplean glosarios terminológicos y se procura mantener la coherencia en una saga, de forma que si los equipos de traducción cambian, la traducción sufra lo mínimo. Lo ideal también es que haya un único editor y coordinador dedicado al proyecto, práctica que suele ser estándar en la localización profesional, excepto en títulos con mucha carga de texto y unos plazos tan ajustados que exigen disponer de dos o más editores. En la traducción amateur, dado el grado de implicación que esto supondría para una sola persona, lo más normal es que haya varios coordinadores y revisores, es decir, se reparte más el trabajo. Esto tiene una ventaja clara, y es que hay una comunicación mucha más fluida de la que suele haber entre ciertos equipos de traductores profesionales, donde muchas veces ni siquiera están en contacto unos con otros y siempre deben pasar por el editor o coordinador en sus conversaciones. Así, aunque aparentemente en la traducción amateur el proyecto esté más disgregado, la realidad es que están más en sintonía, tienen más tiempo, conocen bien el juego y hay mucha más interacción. Por suerte, cada vez se facilita más la comunicación en el equipo. Es de vital importancia poner en contacto a los traductores de un mismo idioma e incluso de otros idiomas, porque las dudas suelen ser comunes a todos, no son exclusivas de una sola lengua, sino que suelen afectar más a la comprensión del original, la dinámica de juego o el argumento. Este debate, que es muy activo y que forma parte intrínseca de la traducción amateur tendría sin duda un efecto muy beneficioso si se extendiese por norma a la traducción profesional.

En la parte técnica, la escena amateur se topa de cara con los mismos escollos con que deben lidiar los profesionales, porque la realidad es que muchos juegos no se preparan para la localización. Las fuentes no incluyen todos los

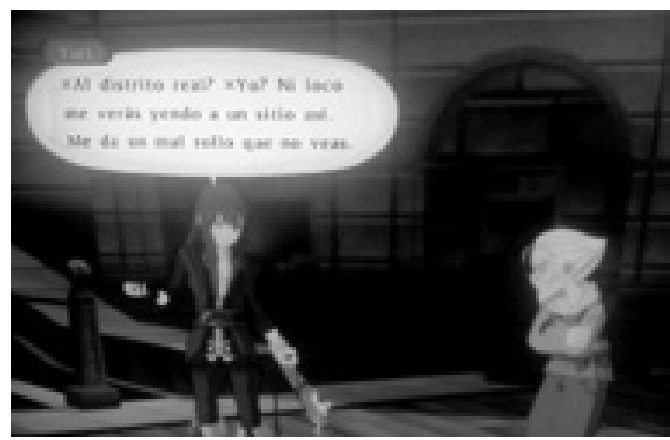

Figura 5. Problemas de caracteres en el Tales of Vesperia (<bttp://notas.tales-tra.com/img/20Io/og/IMG_oogr.jpg $\rangle$ )

caracteres; hay textos incrustados en gráficos que necesitan ser editados para poder traducirlos; el ancho de los menús no está diseñado para que sea flexible y se pueda ampliar o reducir en función del contenido, etcétera.

De ahí que el rombacking, si bien en principio está más orientado a la traducción, tenga un importante componente de modificación técnica, pero solo con el fin único de acomodar los textos traducidos y que se muestren correctamente. Una de las modificaciones habituales consiste en añadir al código símbolos especiales, como el signo de apertura de exclamación e interrogación o caracteres acentuados.

En cuanto a las licencias que se permiten unos y otros a la hora de traducir los juegos, está claro que en el ámbito profesional suele haber una conversación entre el equipo de localización y el cliente, sea este la empresa distribuidora o la desarrolladora, para llegar a un consenso. En ocasiones el cliente es muy estricto, decide limitar el grado de adaptación e impone por su parte una serie de restricciones que el equipo debe acatar. Por ejemplo, puede indicar que no se localicen los nombres de personajes, lugares, objetos, hechizos, etcétera. En esta misma línea, si el cliente no contempla la adaptación de los 
elementos gráficos, especialmente aquellos que contienen textos, el equipo deberá ajustarse al espacio disponible y habrá de procurar que su traducción esté en consonancia con la parte visual, dejando incluso en el idioma original ciertos términos para evitar discrepancias. Así, bien para recortar presupuesto, bien por mantener un mayor control sobre la localización e incluso por miedo a dar carta blanca a los traductores, muchos clientes se muestran especialmente restrictivos y marcan de antemano muchas limitaciones al proceso creativo y de adaptación. La línea entre adaptación y fidelidad al original es borrosa y aunque la mayoría de los jugadores da especial importancia a la segunda, bien es cierto que no siempre es posible, pues si la finalidad de la localización es conseguir la inmersión del jugador y que éste comprenda el juego, el argumento, lo que sucede en pantalla, al dejar en la lengua original ciertos términos se puede perder información, fluidez, naturalidad y, por tanto, inmersión en el mundo virtual. Si bien es cierto que hay ciertos términos en el mundo de los videojuegos que son préstamos, como «goblin» o «doppelgänger», la mezcla de idiomas resulta farragosa en según qué circunstancias y puede interpretarse como errores de traducción o despistes.

\section{¿HÉROES O VILLANOS?}

Tal como recoge el artículo 8 del Convenio de Berna para la Protección de las obras literarias y artísticas, cualquier tipo de traducción realizada por aficionados es ilegal, en tanto que son los autores quienes "gozan del derecho exclusivo de hacer o autorizar la traducción de sus obras» mientras conserven sus derechos sobre la obra original. Así pues, al no estar autorizada, la práctica del rombacking, la traducción y su posterior distribución, es ilegal. Sin embargo, entre la comunidad de rombackers existe la creencia popular de que si solo distribuyen el parche binario, lo que están haciendo es legal, porque los datos son «nuevos». Los parches son archivos creados por ellos, y a eso se aferran. Y sí, es cierto que el contenido del parche está creado por los rombackers, pero el problema es que su contenido es una traducción no autorizada de un contenido protegido. Además, desde su punto de vista, su actividad amateur confunde aún más los límites de la legalidad en tanto en cuanto es una práctica sin ningún ánimo de lucro y que no fomenta la piratería ni forma parte de ella. De hecho, cabe destacar que todos los rombackers tienen un marcado sentido de la moralidad, muchos son coleccionistas y contrarios a la piratería, en prácticamente todos sus sitios web hay notas en contra de esta práctica y siempre instan a los fans a que adquieran copias originales. Tienen prohibido publicar enlaces directos, hospedar o hacer referencia a materiales protegidos por derechos de autor. Por lo que se deduce de sus respuestas, lejos de considerarse unos delincuentes se ven más como una suerte de Robin Hood que reparte entre los fans la esperada traducción de esos juegos con los que tanto disfrutan. Por suerte para ellos, la práctica del rombacking en lugar de afectar negativamente a las ventas es una excelente forma de dar a conocer ciertos títulos y de alargar su vida natural y posiblemente por eso las empresas no han tomado cartas en el asunto. De momento.

\section{EL ROMHACKING, ¿LA MEJOR PUBLICIDAD?}

Cuando se pregunta a los rombackers cómo creen que pueden afectar sus prácticas a la industria, todos están de acuerdo en que sus traducciones aportan valor al juego, simplemente porque llega a más gente que no lo jugaría 
si no estuviera en su idioma. Recalcan además que no hay ningún ánimo de lucro por su parte, se muestran contrarios a la piratería, defienden siempre la compra de copias originales y demuestran una actitud bastante responsable en este sentido. La comunidad lo tiene claro: si los juegos estuviesen localizados, no existiría el rombacking y consideran que las propias desarrolladoras y distribuidoras se hacen daño a sí mismas al no localizar a los idiomas más hablados y leídos del mundo. La realidad es que son muchos los jugadores que no adquieren un título porque no está disponible en su idioma; prueba de ello son los innumerables comentarios al respecto que se vierten en los foros en Internet.

\section{Campañas online}

Cada vez que las distribuidoras anuncian que los títulos que los fans llevan meses o incluso años esperando, con las expectativas por las nubes, no llegará traducido a su idioma, se sienten decepcionados. Los rombackers, por contar con un buen nivel de inglés (de ahí que se animen a traducir), son los menos activos en este sentido, pues ellos a título individual no tienen problema en comprarse el juego y disfrutarlo en inglés (en proporción son menos los títulos originales japoneses que ni siquiera se localizan al inglés). Solo en los casos más extremos, cuando las distribuidoras anuncian que un título estará localizado al español y en el momento del lanzamiento se descubre que no es así, algunos rombackers sí toman cartas en el asunto y apoyan con sus firmas o comentarios las peticiones online que organizan los jugadores. Aunque lo más común es que directamente se pongan manos a la obra y centren sus esfuerzos en localizar ellos mismos el juego.

Publicaciones online, como meristation. com - la revista online de videojuegos más

\section{Tales of Vesperia, sin traducir $\mathbf{y}$ en completo inglés en España Los jugadores protestan en los foros y piden publicamente una solución alternativa.}

Figura 6. Titular de Meristation (<http://www. meristation.com/ $/ v_{3} /$ des_noticia.php? $i d=c w_{4} a_{43} 29 a 189 e a 2$ Eppic $=G E N>$ )

leída en español- suelen hacerse eco de las acciones que ponen en marcha los fans, como las plataformas para conseguir la localización de un videojuego. Una de esas plataformas es la de $I C O$, que logró que Sony reeditara este juego en España, o la plataforma $M G S_{3}$ Subsistence, que también consiguió su objetivo de que Konami lanzara en España la versión especial de Metal Gear Solid 3. Otras, como la de Okami no tuvieron tanta suerte. Pero eso sí, traducido está, por Reservoir Wolves.

\section{CROWDSOURCING, LA TERCERA VÍA}

Atrás queda ya la época en que los videojuegos eran considerados como un producto físico: el jugador entraba en una tienda y compraba un cartucho o un $\mathrm{CD}$ que después reproducía en casa, en su PC, consola o dispositivo portátil. Con la evolución del videojuego y el apogeo de las redes sociales han surgido nuevos formatos de distribución como la descarga online, y nuevas formas de juego, derivadas de la llamada Web 2.0 en que los propios usuarios generan contenidos sobre la marcha. Esta constante actualización de los contenidos exige un nuevo modelo de localización. El crowdsourcing o «subcontratación voluntaria» puede ser la respuesta para asumir la gran cantidad de textos que se generan, sin disparar los costes de localización. Este sistema parece haber funcionado relativamente bien para que Wikipedia, Face- 
book, Twitter o juegos online como eRepublik tradujeran sus menús y documentos de ayuda.

Pero el crowdsourcing presenta una serie de «peligros», sobre todo por el tamaño de las comunidades a las que se confían las tareas de traducción. Debería entenderse como una tercera vía, una combinación entre la traducción amateur, hecha por y para los fans, con las ventajas de familiarización y motivación que esta conlleva; y la traducción profesional, organizada, con editores y coordinadores experimentados y un sistema de gestión a la altura de la tarea. Si se da total libertad a los usuarios para abordar la traducción, el resultado será caótico. Las comunidades online cuentan a sus miembros por miles, decenas de miles, millones. $\mathrm{Si}$ en la escena amateur, los grupos sienten la necesidad de limitar el número de personas que participan en el proyecto y de organizarlas de forma eficaz, con mayor razón deberían fijarse unos límites al recurrir al crowdsourcing. Es entonces donde entra la parte profesional; las empresas han de aplicar una serie de prácticas para garantizar la calidad del producto.

\section{CONCLUSIONES}

Es un mito y una falacia pensar que todos los jugadores tienen un nivel de inglés lo bastante bueno como para disfrutar de un videojuego en ese idioma y el buen estado de salud de que goza la traducción amateur en el mundo hispano es prueba de ello. Esta práctica nace de la devoción que sienten los jugadores por ciertos títulos y de su frustración al descubrir que no llegarán a sus respectivos países o que no estarán disponibles en su idioma. En esta última década la comunidad ha madurado, se ha ido organizando y los proyectos cada vez guardan mayor parecido con las localizaciones profesionales, tanto en la metodología como en la calidad del resultado final, si bien los límites temporales son mucho más relajados. En la escena profesional, factores como la confidencialidad, la premura y lo ajustado de los plazos juegan claramente en contra de la calidad final. Los traductores e ingenieros aficionados están estrechamente conectados al tejido «fan», forman parte de él y ese es su principal punto fuerte. Ellos ponen de manifiesto que la familiarización es vital para conseguir un trabajo excelente y que los desarrolladores siguen considerando la localización como un proceso más de la post-producción, cuando si se planificase desde la concepción y el diseño y estuviese presente durante el desarrollo, se ahorraría tiempo de traducción y, sobre todo, de testeo. Entretanto, podemos afirmar sin miedo que la comunidad hispana le está muy agradecida a estos traductores e ingenieros amateurs que siguen en activo y muy motivados.

\section{AGRADECIMIENTOS}

Quisiera dar las gracias a Miguel Á. BernalMerino por sus comentarios y correcciones, así como a la colaboración desinteresada de los rombackers que atendieron mis preguntas y consultas. Este artículo está dedicado a ellos: Hexplus, Cyclops, DaRKWizardx, PacoChan, Soyzwiz, Reinhardt, Wastor y Pablito's.

RECIBIDO EN OCTUBRE DE 2 OIO

ACEPTADO EN ENERO DE $2 O I I$

VERSIÓN FINAL DE FEBRERO DE $2 O I I$

\section{REFERENCIAS ELECTRÓNICAS}

Clan Dlan, <http://www.clandlan.net/> [Consulta: 6 de julio de 2009]

Convenio de Berna para la protección de las obras literarias y artísticas, <http://www.wipo.int/treaties/es/ip/berne/trtdocs_wooor.html> [Consulta: 6 de julio de 2009] 
Fortaleza Romhack, <http://fortaleza.romhackhispano.org/> [Consulta: 3 de julio de 2009]

InfoEmuladores, «Entrevista a ChronoSP Team» (2009), <http://www.info-emuladores.es/index. php?seccion=entrevistas\&entrevista $=$ chronosp > [Consulta: 5 de octubre de 20Io]

Muñoz Sánchez, Pablo (2008). «En torno a la localización de videojuegos clásicos mediante técnicas de rombacking: particularidades, calidad y aspectos legales», JoSTrans, The Journal of Specialised Translation $9: 80-95$

$<$ http://www.jostrans.org/issueog/art_munoz_sanchez.php> [Consulta: 3 de julio de 2009]

Mushroom Coorporation, «El rom hacking» (2006) <http://www.mushroomcorporation.com/?p=I2> [Consulta: 3 de julio de 2009]

Plataforma ICO, <http://www.plataforma-ico.esp. st/> [Consulta: 8 de octubre de 20Io]

Plataforma MGS 3 Subsistence, <http://www.plataformamgs3s.ya.st/> [Consulta: 8 de octubre de 20IO]

Reservoir Wolves, Okami, <http://okami.romhackhispano.org/\#contenido $>$ [Consulta: 8 de octubre de 20Io]

Romhacking.net, <http://www.romhacking.net/> [Consulta: ro de julio de 2009]

Tales Translations, <http://blog.tales-tra.com/> [Consulta: 5 de octubre de 2009]

Último Nivel, Entrevista a Clan DLAN, <http:// www.ultimonivel.net/2009/r2/entrevista-a-clandlan/> [Consulta: 5 de octubre de 20Io]

Wikipedia, Fan Translation of Video Games http:// en.wikipedia.org/wiki/Fan_translation_of_ video_games [Consulta: 3 de julio de 2009]

\section{REFERENCIAS GRÁFICAS}

<http://blog.tales-tra.com/2009/noticias/sobre-latraduccion-de-tales-of-vesperia> [Consulta: 5 de octubre de 20Io]

$<$ http://blog.tales-tra.com/proyectos/tales-of-vesperia> [Consulta: 7 de octubre de 2010]

<http://devil.romhackhispano.org/lunar.html> [Consulta: 7 de octubre de 20Io]

<http://notas.tales-tra.com/img/201o/og/amerigobient-690x388.jpg> [Consulta: 5 de octubre de 20IO]

<http://notas.tales-tra.com/img/201o/og/ IMG_0o9I.jpg> [Consulta: 6 de octubre de 20Io] <http://notas.tales-tra.com/img/20ro/og/ IMG_0094.jpg > [Consulta: 6 de octubre de 2010]

<http://okami.romhackhispano.org/img/translhexion.jpg> [Consulta: 6 de octubre de 20Io]

$<$ http://toa.tales-tra.com/i/manual-explicacion-3. png> [Consulta: 6 de octubre de 20Io]

<http://www.meristation.com/v3/des_noticia. php?id $=$ cw4a4329ar8gea2 $\&$ pic $=\mathrm{GEN}>$ [Consulta: 6 de octubre de 2010] 